\title{
Editorial Message: Special Issue on Innovative Fuzzy Systems for Ergonomics in Real-World Settings
}

\author{
Li-Wei Ko ${ }^{1}$
}

Published online: 24 November 2021

(C) Taiwan Fuzzy Systems Association 2021

Ergonomics is the scientific discipline concerned with understanding the principles underlying interactions between humans and other elements of a system, and the profession that applies these principles and understanding to designs in order to optimize human well-being and overall system performance. As human behavior is always dynamic, making it challenging to predict and access, it is worth applying fuzzy theories and control systems with intelligent computational technologies to enhance the interaction performance between humans and the systems. The main purpose of this special issue is to understand how innovative fuzzy system and algorithm developments relate to and enhance human-machine interaction in real-world settings. This special issue was organized in the 2020 International Conference on Fuzzy Theory and Its Applications (iFUZZY 2020) held at National Chiao Tung University, Hsinchu, Taiwan, on November 4-7, 2020. The invited guest editors of the special issue are Prof. Li-Wei Ko, Prof. I-Fang Chung, Prof. Tzyy-Ping Jung, Prof. ChinTeng Lin, and Prof. Jiali Yu.

In this special issue, we included 8 high-quality papers out of 15 submissions from four different research fields. These papers have been published in the International Journal of Fuzzy Systems after the elaborative revisions based on the technical reviewers' precious and valuable comments and suggestions. Based on the research scopes of the accepted papers, we categorized the main focus of these papers to four different topics: (1) Innovative Fuzzy System Development and Optimization, (2) Innovative

Li-Wei Ko

liwei.ko@nycu.edu.tw

1 National Yang Ming Chiao Tung University, Hsinchu, Taiwan
Fuzzy Systems/Algorithms for Exoskeleton Robot Control and Human-Machine Interaction, (3) Innovative Fuzzy Systems/Algorithms for Brain-Computer Interfaces, and (4) Innovative Fuzzy Systems/Algorithms for Management and Assessment. We briefly introduce each paper as follows, to offer the readers a better idea of the kind of works that form this special issue.

In the topic of Innovative Fuzzy System Development and Optimization, $\mathrm{Wu}$ et al. proposes the multiobjective $\mathrm{H} 2 / \mathrm{H} \infty$ fuzzy control design problem for nonlinear stochastic chaotic systems, and obtains the desired robustness and optimization in Pareto optimal sense. The T-S fuzzy model and the fronts-squesszing linear matrix inequalities constrained multiobjective evolution algorithm are introduced to overcome the multiobjective $\mathrm{H} 2 / \mathrm{H} \infty$ fuzzy control design problem. This paper demonstrated a dynamic constrained multiobjective optimization problem (MOP) into a MOP constrained by linear matrix inequalities (LMIs) and MOP constrained by LMIs.

In the topic of Exoskeleton Robot Control and HumanMachine Interaction, there are two papers included in this field. First, Huang et al. proposed an assistive robot design for lower limbs rehabilitation using fuzzy control. In order to keep the patient's shank horizontal when the knee joint is flexed, they used discrete-time fuzzy control methodology to design a controller for the stability of the robot system. The preliminary experimental results show the effectiveness of rehabilitation of stroke patients using this assistive robot. This is a very valuable study in lower limbs rehabilitation. Second, Lee et al. integrated hardware design and Interval Type II theory to complete the implementation of a Mobile Over-Ground Gait Training System. This system rejuvenates exercise their lower limb appropriately to slow down motor function degradation, rebuild or strengthen the subject's motor function. Finally, based 
on the interval type-2 fuzzy sliding control methodology and pulse-width modulation (PWM) technology, this paper demonstrated that the mobile-OGTS provides a stable control force to assist subjects in over-ground gait training and gives assist-as-needed body weight support system (BWSS) during gait training.

In the topic of Brain-Computer Interfaces applications, there are two papers that presented the motor imagery EEG classification and mild cognitive impairment EEG-based classification using fuzzy classifier. The first paper is Lin et al. adopted the ensemble model to improve the classification accuracy across participants in the motor imagery of left and right hands. The ensemble method combines the linear discriminant analysis (LDA), support vector machine (SVM), and fuzzy twin SVM (FTSVM) classifier, in which the fuzzy classifier can reduce the influence of artifacts. The paper finally shows that the performance of the ensemble model that includes the FTSVM classifier is superior to the ensemble model that without using the FTSVM classifier. The second paper is Hsiao et al. described a kernel-based fuzzy support vector machine (CKF-SVM) with features from sample entropy (SampEn) and Katz's fractal dimension (KFD), to classify individuals with mild cognitive impairment (MCI) patients and healthy controls (HC) based on resting-state EEG data. By recording two 90-s long EEG data, they were already able to achieve $90.19 \%$ accuracy in MCI-HC classification, and the CKF-SVM outperforms LDA, QDA, k-NN and SVM in MCI classification tasks.

In the topic of Management and Assessment, there are three papers included in this field. These papers are focusing on the application of the innovative fuzzy approach for management and assessment. The first paper is that Lin et al. proposed a fuzzy-DEMATEL approach to improve the service quality of wealth management banks for high-net-worth customers during the 2019-2020 COVID-19 pandemic in Taiwan. They used four dimensions of surveys and interviews including bank performance, service quality, customer relations, and COVID-19, and used the fuzzy language scale to obtain their expert opinions from 10 bank executives. This paper showed that the most important factors were investment information, conversion behavior, cost income, and the number of confirmed cases of COVID-19 (the top five major affected countries). The second paper is that Huang et al. integrated the neutrosophic set on the basics of the DEMATEL methods, to explore the key factors that prevent the spread of epidemics when knowledge is incomplete. The results show that the combination of neutrosophic set theory leads to more meaningful evaluation under incomplete information. This study can help the government understand the causal relationship between factors and the development of scientific decision-making. The third paper is that Bae et al. proposed a new happiness model based on fuzzy external force on the basis of Sprott's Happiness Model and SpringDenter-mass Model. At the same time, the typical chaotic path behavior, including periodic motion (getting large euphoria status), chaotic motion (euphoria approach maximum status), and periodic window (eventually euphoria will decrease and become calm), is expressed by the simulation of parameter assignment.

Studying through the above papers, researchers can better understand how innovative fuzzy system and algorithm development relate to human-computer interaction and various thematic applications. In addition, there are also contributions to the theoretical optimization with new concepts and models of fuzzy application. We envision that the papers of the special issue will be of great interest to researchers and students of the innovation fuzzy systems and human-computer interaction areas, as well as in different application areas.

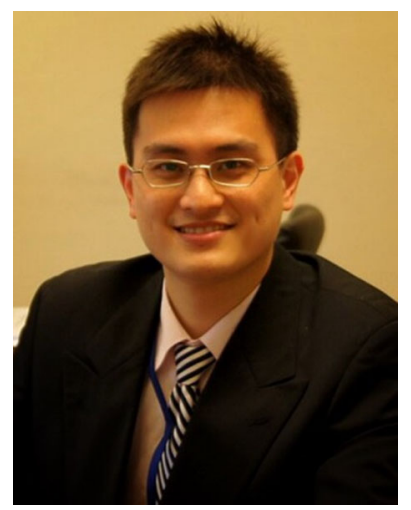

Li-Wei Ko is with the Institute for Bioinformatics and Systems Biology and Department of Electrical and Computer Engineering, National Yang Ming Chiao Tung University, Hsinchu 30010, Taiwan. He is also with the Center for Intelligent Drug Systems and Smart Bio-Devices $\left(\right.$ IDS $\left.^{2} \mathrm{~B}\right)$ and Brain Research Center in National Yang Ming Chiao Tung University, Hsinchu 30010, Taiwan. He is also an adjunct professor in the Drug Development and Value Creation Research Center, Kaohsiung Medical University, Kaohsiung 80708, Taiwan. 